\title{
Water-soluble fullerene (C60) inhibits the osteoclast differentiation and bone destruction in arthritis
}

This article was published in the following Dove Press journal:

International Journal of Nanomedicine

19 October 2009

Number of times this article has been viewed

\author{
Kazuo Yudoh' \\ Rie Karasawa' \\ Kayo Masuko² \\ Tomohiro Kato ${ }^{2}$ \\ 'Department of Frontier Medicine, \\ 2Department of Biochemistry, \\ Institute of Medical Science, \\ St. Marianna University School \\ of Medicine, Kawasaki, Japan
}

Correspondence: Kazuo Yudoh Dept. of Frontier Medicine, St. Marianna University School of Medicine 2-16-I Sugao, Miyamae-ku, Kawasaki City 216-85I2, Japan

Tel +8I 44977 8I I I (ext. 4209)

Fax +8I 449782038

Email yudo@marianna-u.ac.jp

\begin{abstract}
Recently, it has been demonstrated that oxygen free radicals have an important role as a signaling messenger in the receptor activator NFKB (RANK) signal pathway required for osteoclast differentiation. The aim of this study was to examine the potential of a strong freeradical scavenger, water-soluble fullerene (C60), as a protective agent against the RANK-induced osteoclastogenesis and osteoclastic bone destruction in arthritis, both in vitro and in vivo. The effects of $\mathrm{C} 60$ on the RANK-induced osteoclastogenesis and osteoclastic bone resorption were examined in vitro. Adjuvant-induced arthritic rats were used as an animal model of arthritis. Rats were divided into two subgroups: control and treatment with $\mathrm{C} 60$ at $1.0 \mu \mathrm{M}$. The left ankle joint was injected intra-articularly with water-soluble C60 (20 $\mu 1)$ in the C60-treated group, while, as a control, the left ankle joint in the control rats received phosphate-buffered saline $(20 \mu 1)$ once weekly for eight weeks. Ankle joint tissues were prepared for histologic analysis. C60 significantly inhibited the responses of osteoclast precursor cells to RANK ligand, including osteoclast differentiation and osteoclastic bone resorption in vitro. In adjuvant-induced arthritic rats, intra-articular treatment with $\mathrm{C} 60$ in vivo reduced the number of osteoclasts and alleviated bone resorption and destruction in the joints, while control ankle joints showed progression of joint destruction with time. These findings indicate that $\mathrm{C} 60$ downregulates the RANK-induced osteoclast differentiation and is a potential therapeutic agent for inhibition of osteoclastic bone destruction in arthritis.
\end{abstract}

Keywords: fullerene, osteoclast, bone resorption arthritis, antioxidant

\section{Introduction}

Rheumatoid arthritis (RA) is a chronic inflammatory disease characterized by synovitis involving synovial hyperplasia with neoangiogenesis and infiltration of lymphocytes and macrophages into the synovial tissue. ${ }^{1,2}$ Synovial hyperplasia erodes and destroys the bone and cartilage in the joint tissues of RA patients. In the joint of patients with RA, osteoclastic hyper-resorption is accelerated as the disease progresses. ${ }^{3}$ Recently, it was found that osteoimmunology, shared mechanisms, and crosstalk between the immune system and bone metabolism are closely involved in the pathogenesis of RA. ${ }^{4-7}$ Induction of osteoclast differentiation from precursor cells is known to be linked by receptor activator of NFKB (RANK)-RANK ligand (RANKL) signals. ${ }^{6}$ When RANKL binds to RANK in an osteoclast precursor cell, RANKL-RANK signals are conveyed downstream to $\mathrm{NF} \kappa \mathrm{B}, \mathrm{P} 38$, and others, provoking the induction of differentiation to osteoclasts. ${ }^{5,6}$ RANKL is expressed by synovial fibroblasts, activated T cells, and macrophages as well as osteoblasts and the expression is induced by proinflammatory cytokines including interleukin-1 (IL-1), IL-6, IL-17, and tumor 
necrosis factor- $\alpha$ (TNF- $\alpha$ ), which are implicated in the pathogenesis of RA. ${ }^{6-8}$

A series of recent reports have indicated that reactive oxygen species (ROS) play a critical role in osteoclast differentiation initiated by RANK-RANKL signals. ${ }^{9-13}$ It has been already reported that several of the intracellular signals essential for osteoclast formation, including NFKB, c-Jun aminoterminal kinase, PI3-kinase, and p38 MAPK, are sensitive to ROS. ${ }^{9-11}$ Osteoclast formation has been shown to depend on $\mathrm{NF} \kappa \mathrm{B}$ activation. ${ }^{7,9,13}$ It has been demonstrated that $\mathrm{NF} \kappa \mathrm{B}$ is a target for cellular activation by $\mathrm{ROS}^{9,12}$ and that antioxidants suppress NFKB activation in osteoclasts. ${ }^{13-15}$ Recent studies have clearly revealed that ROS mediates RANK-RANKL signaling in osteoclastogenesis. ${ }^{11-14}$ Lean and colleagues demonstrated that estrogen deficiency causes osteoclastic hyper-resorption by lowering antioxidant defenses in osteoclastic cells, thus entraining a ROS-mediated increase in osteoclastic differentiation and bone resorption. ${ }^{9}$ In addition, it has been demonstrated that systemic administration of antioxidants prevented the loss of bone that normally occurs with time in animal models. ${ }^{15}$ Therefore, targeting of ROS could have a distinct therapeutic value as a strategy to prevent osteoclastic hyper-resorption in arthritis.

Fullerene (C60) is composed of spherical carbon molecules with a unique cage structure. ${ }^{16,17}$ This compound has a high reactivity with oxygen free radicals and potential activity as a strong free-radical scavenger. ${ }^{18}$ It has been reported that the antioxidant level of $\mathrm{C} 60$ is several hundredfold higher than that of other antioxidants. ${ }^{18}$ Recently, C60 derivatives have shown some remarkable biologic properties such as neural apoptosis, ${ }^{19}$ promotion of chondrogenesis, ${ }^{20}$ and protection of skin keratinocytes from ROS-induced cell death after ultraviolet stress. ${ }^{21,22}$ We have already demonstrated that water-soluble C60 fullerene, a strong free-radical scavenger, can function as a protective agent against catabolic stress-induced degeneration of articular cartilage in a model of osteoarthritis in vitro and in vivo. ${ }^{23}$ Water-soluble C60 significantly reduced articular cartilage degeneration in the osteoarthritis rabbit model. These findings suggest that $\mathrm{C} 60$ has the potential to protect against oxygen free radical-induced pathological features in a variety of diseases. Exploitation of C60 could lead to the development of novel therapeutic strategies in the prevention of both inflammation/synovitis and osteoclastic hyperresorption in RA.

In the present study, we investigated the influence of water-soluble $\mathrm{C} 60$ on generation of osteoclast differentiation and osteoclastic bone resorption in order to explore any therapeutic or protective effect of C60 on the bone hyper-resorption in arthritis. We confirmed the suppressive effects of $\mathrm{C} 60$ on the RANK-induced osteoclast differentiation and osteoclastic bone resorption in vitro. Our results indicated that intra-articular treatment with C60 significantly reduced bone resorption and joint destruction in adjuvantinduced arthritic rats. These findings demonstrate that C60 has suppressive effects on osteoclastic bone resorption and the resultant joint destruction in arthritis, suggesting that it could be useful for prevention and treatment of arthritis.

\section{Patients and methods}

\section{Effect of $\mathrm{C} 60$ on bone resorption in vitro}

In arthritis, especially in RA, bone destruction progresses with advancing inflammation. We therefore examined whether C60 suppressed osteoclast differentiation and osteoclastic bone resorption in vitro. According to two very specific markers of authentic osteoclasts, we have thus defined multinucleated (more than or equal to three nuclei) tartrateresistant acid phosphatase (TRAP)-positive cells as mature osteoclasts, which could form resorption pits on dentine slices, verifying these cells as functional osteoclasts.

\section{Influence of C60 on RANK-induced osteoclast differentiation in vitro}

Poietic human osteoclast precursor cells were purchased from BioWhittaker Inc (Walkersville, MD, USA). To induce osteoclast differentiation in vitro, the osteoclast precursor cells were plated at a density of $5 \times 10^{4}$ cells per well in a final volume of $0.4 \mathrm{ml}$, using eight well chamber slides (Nalge Nunc International Corp, Naperville, IL, USA) and cultured in $\alpha$-MEM supplemented with $10 \%$ heat-inactivated fetal calf serum (FCS), penicillin $(50 \mathrm{U} / \mathrm{ml})$, streptomycin $(50 \mathrm{mg} / \mathrm{ml})$, and $33 \mathrm{ng} / \mathrm{ml} \mathrm{M-CSF}$, at $37{ }^{\circ} \mathrm{C}$ in $5 \% \mathrm{CO}_{2} / 95 \%$ air, in a humidified incubator. The cultures were further stimulated with $30 \mathrm{ng} / \mathrm{ml}$ soluble RANKL in the presence or absence of C60 (Vitamin C60 Inc., Tokyo, Japan; 0.1, 1.0 , or $10.0 \mu \mathrm{M})$. The cells were fed by replacing half the medium twice weekly with fresh stimuli. After seven days of culture, cells were fixed and stained for TRAP, using a commercial kit (Sigma, St. Louis, MO, USA) according to the manufacturer's instructions.

TRAP staining was carried out as follows. After the incubation period, the medium was removed and wells were washed with $100 \mu \mathrm{L}$ phosphate-buffered saline (PBS). Fixing solution $(50 \mu \mathrm{L})$ was then added and the plate was centrifuged at room temperature for five minutes. After removing the fixing solution, the wells were washed 
with $250 \mu \mathrm{L}$ distilled diluted water (DDW) three times. Chromogenic substrate solution $(50 \mu \mathrm{L})$ was added to each well and the plate was incubated at $37{ }^{\circ} \mathrm{C}$ for 20 minutes. When appropriate color developed, the chromogenic substrate solution was removed and the reaction was terminated in each well with $250 \mu \mathrm{L}$ DDW. The wells were washed with DDW three times, then the surround was removed and the cells were mounted in water-soluble mounting agent (NUNK 178599). The number of TRAP-positive multinucleated giant cells/field was counted under reflected light using a digital high fidelity microscope VH-8000 (x 200, Keyence Co, Ltd, Osaka, Japan).

\section{Effect of $\mathrm{C} 60$ on bone resorption by osteoclasts}

The ability of osteoclasts to resorb pits was assessed by generating mature osteoclasts on ivory dentine slices (Wako Co, Ltd, Osaka, Japan) in 96 well plates. Osteoclast precursor cells were incubated in the presence or absence of $\mathrm{C} 60(0.1,1.0$, or $10.0 \mu \mathrm{M})$. After 14 days of culture, dentine slices were washed with phosphatebuffered saline (PBS) and incubated for 18 hours in $0.25 \mathrm{M}$ ammonium hydroxide, followed by sonication in PBS for 30 seconds. Dentine slices were washed with PBS and stained in Mayer's hematoxylin solution for two minutes, and pits were photographed under reflected light with a digital high fidelity microscope (VH-8000). The total pit areas were measured in four randomly selected areas of each dentine slice using an image analysis system (Image Gauge version 4.0, Fuji Photo Film Co, Ltd, Tokyo, Japan).

\section{Efficacy of $\mathrm{C} 60$ in animal models of arthritis}

We investigated whether $\mathrm{C} 60$ had any suppressive effects on arthritis (synovitis and joint destruction) using a rat arthritis model. Eight-week-old female SD rats were obtained from Charles River Laboratories Japan (Tokyo, Japan). Adjuvantinduced arthritic rats were created as described in a previous report. ${ }^{24}$ The ankle joints of 40 rats were assessed. After being once immunized to produce arthritis, the rats were divided into a control group ( $\mathrm{n}=20$ rats) and a C60-treated group $(\mathrm{n}=20$ rats). In the control group, the left ankle joint was treated with an intra-articular injection of $20.0 \mu \mathrm{L}$ PBS once per week for eight weeks, using a micro-needle syringe. In the C60-treated group, the left ankle joint was similarly treated with an intraarticular injection of $20.0 \mu \mathrm{L} \mathrm{C60}(10.0 \mu \mathrm{M})$ once per week for eight weeks. The right knee joints were not treated in either group to be compared to the contralateral ankle joints for the observation of arthritis development and progress.
The rats were euthanized at four and six weeks ( $n=4$ rats) after immunization and both ankle joints were harvested. Bone and cartilage tissue blocks were prepared for histological analysis. All experiments were conducted with the approval of the University Animal Care and Use Committee.

Articular bone and cartilage samples were fixed for two days in 4\% paraformaldehyde solution and then decalcified in $4 \%$ paraformaldehyde containing $0.85 \%$ sodium chloride and $10 \%$ acetic acid. Tissues were dehydrated through a series of ethanol solutions and infiltrated with xylene before being embedded in paraffin and cut into $6-\mu \mathrm{m}$ sections. Sections were deparaffinized through sequential immersion in xylene and a graded series of ethanol solutions in accordance with conventional procedures. The number of osteoclastic multinucleated giant cells/field was counted in six randomly selected bone areas in each sample under reflected light using a digital high fidelity microscope (VH-8000). Three independent observers assessed bone damage in a blinded manner.

In addition, the histological characteristic of bone tissue was scored by three blinded observers. Bone destruction was graded from 0 to 3 , where $0=$ normal, $1=$ mild loss of cortical bone at few sites, $2=$ moderate erosion of cortical and trabecular bone, and $3=$ marked destruction of bone at many sites. Three independent observers assessed bone damage in a blinded manner. ${ }^{25}$

\section{Statistical analysis}

Results are expressed as means \pm standard deviations. Data were analyzed by a nonparametric statistical analysis. A $P$ value $<0.05$ was considered statistically significant.

Student's $t$-test was used to assess the differences between C60-treated and control groups with respect to arthritis and histologic parameters. When a statistically significant difference between the two groups was detected, analysis of variance (ANOVA) was used to adjust for the confounding effects of animals and observers.

\section{Results and discussion C60 inhibits osteoclast differentiation}

To examine the inhibitory effect of C60 on osteoclast differentiation, the TRAP activity of the cells was observed during the differentiation of osteoclast precursor cells into osteoclasts. As shown in the representative images (Figure 1A), C60 at $10.0 \mu \mathrm{M}$ inhibited the differentiation of osteoclast precursor cells into TRAP-positive multinucleated giant cells. There was a significant difference in the 
A

Control

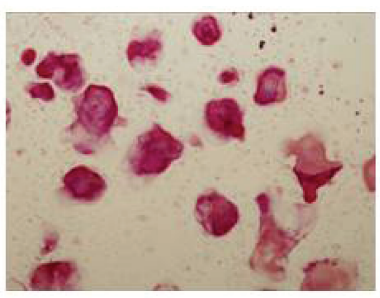

C60: $0.1 \mu \mathrm{M}$

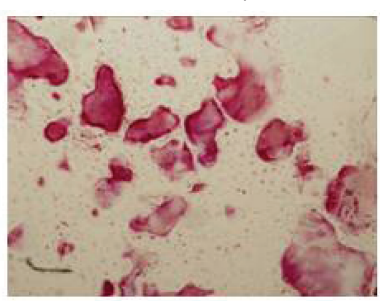

C60: $1.0 \mu \mathrm{M}$

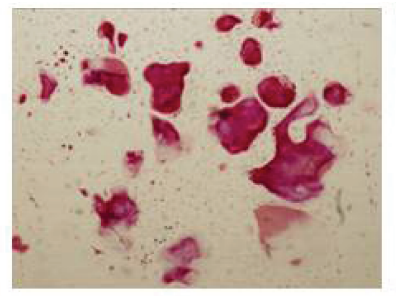

C60: $10 \mu \mathrm{M}$

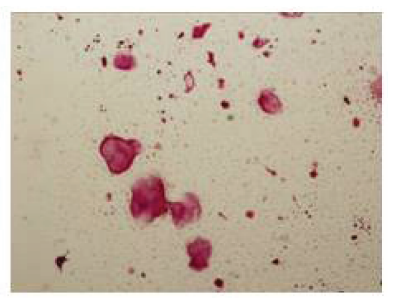

B

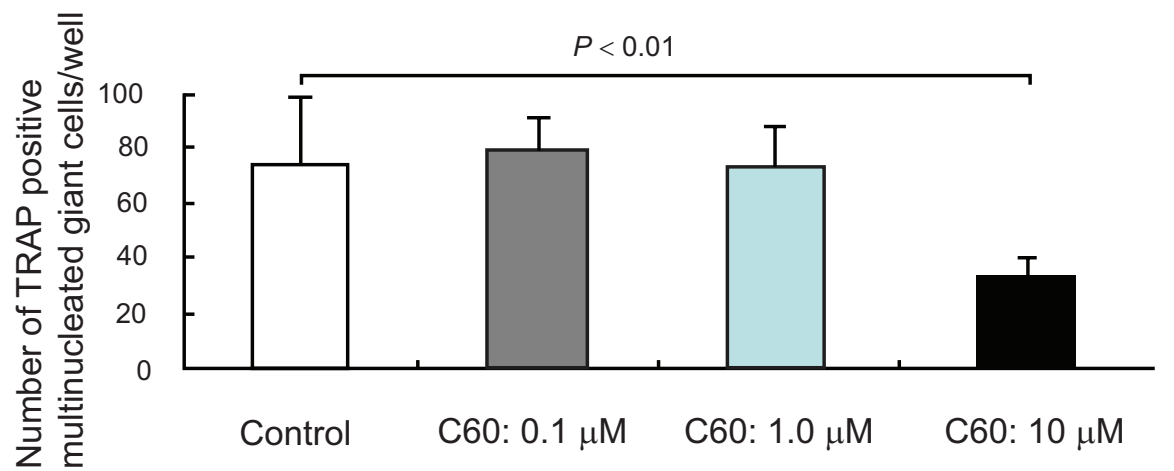

Figure I Inhibitory effects of $\mathrm{C} 60$ on the osteoclastogenesis. Osteoclast precursor cells were incubated with osteoclast differentiation factors in the presence or absence of water-soluble $\mathrm{C} 60(0.1,1.0$, or $10.0 \mu \mathrm{M})$ for seven days. Data from four independent experiments were analyzed. A) At the end of incubation period, the osteoclastogenesis was analyzed by the tartrate-resistant acid phosphatase (TRAP) staining. Representative images $\mathbf{A}$ ) showed that $\mathrm{C} 60$ at $10.0 \mu \mathrm{M}$ inhibited the differentiation of osteoclast precursor cells into TRAP-positive multinucleated giant cells. B) There was a significant difference in the TRAP positivity between the control group and the I0.0 $\mu$ M C60-treated group $(P<0.01$ compared to the control).

TRAP positivity between the control group and the $10.0 \mu \mathrm{M}$ C60-treated group (Figure 1B).

\section{C60 inhibits osteoclastic resorption}

The activity of osteoclasts was assessed by examining their ability to form resorption lacunae on dentine slices. As shown in Figure 2A, C60 at 1.0 and $10.0 \mu \mathrm{M}$ reduced the bone resorption area formed by the cells. There were significant differences in bone resorption between the control and the C60-treated groups (Figure 2B; $P<0.01: 0.1 \mu \mathrm{M}$ C60-treated group, $P<0.01: 1.0 \mu \mathrm{M}$ C60-treated group).

Also, we performed the experiment to verify whether C60 influenced the bone resorping activity of mature osteoclasts in vitro. After the maturation of osteoclasts, the pit formation assay was performed in the presence or absence of C60. As a result, in spite of the presence or absence of 660 , there was no significant difference in the pit formation by the C60-treated mature osteoclasts in comparison with the control (mature) osteoclasts in the assay (data not shown). C60 did not influence the mature osteoclast activity, although C60 suppresses the differentiation of osteoclasts from the precursor cells. From the results, we concluded that inhibitory effect of
C60 resulted from not the suppression of osteoclast activity but the reduced number of mature osteoclasts.

\section{Pathological features of the arthritic rat model}

Adjuvant-induced arthritic rats were administered C60 $(10.0 \mu \mathrm{M})$ once per week by intraarticular injection into the ankle joints during the development of arthritis. The severity of arthritis was monitored every two weeks after immunization. In the control joints, at the four-week and six-week time points, histologic features of arthritis were evident. These joints exhibited cartilage degeneration (extension of the area of surface fibrillation, chondrocyte clustering, abnormal deposition of chondrocytes and a decreased cell density with cartilage thinning), synovial hyperplasia with neoangiogenesis, infiltration of inflammation-related cells into the synovial tissue, and bone resorption/destruction by multinucleated osteoclastic cells of the subchondral bone and areas of exposed bone. In contrast, treatment of the ankle joints with C60 markedly inhibited synovitis, such as synovial hyperplasia and infiltration of inflammation-related 

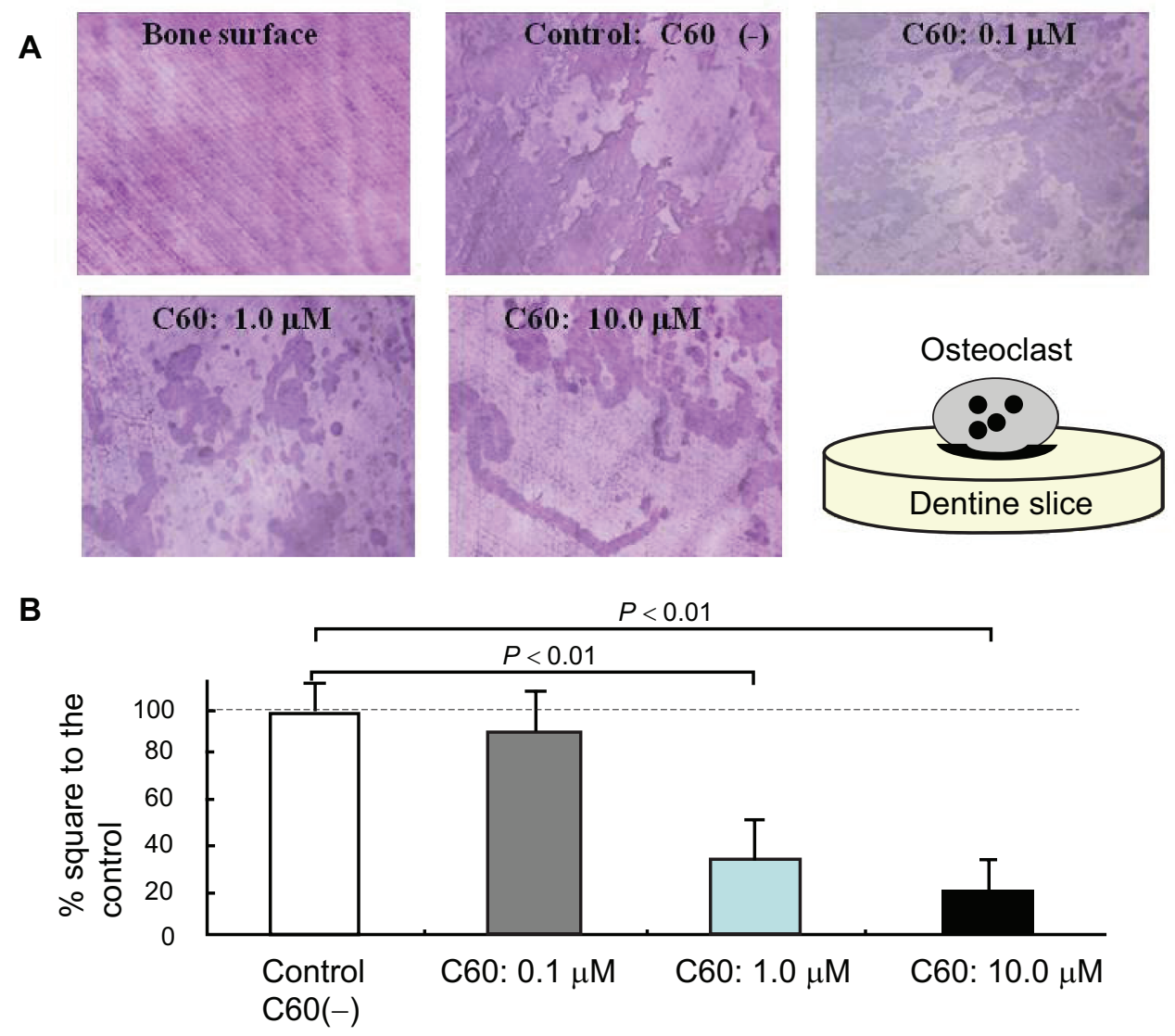

Figure 2 Inhibitory effect of $\mathrm{C} 60$ on osteoclastic bone resorption in vitro. A) Osteoclast precursor cells were incubated with osteoclast differentiation factors in the presence or absence of $\mathrm{C} 60(0.1,1.0$, or $10.0 \mu \mathrm{M})$. At the end of the incubation period, the ability of osteoclasts to resorb pits was assessed by the total pit areas on ivory dentine slices. Data from four independent experiments were analyzed. As the representative images, $\mathrm{C} 60(1.0$ and $10.0 \mu \mathrm{M})$ inhibited the pit formation resorted by the cells. B) There were significant differences in bone resorption between the control and the C60-treated groups $(P<0.01$ : I.0 $\mu \mathrm{M}$ C60-treated group, $P<0.0$ I: I0.0 $\mu \mathrm{M}$ C60-treated group).

cells, and joint destruction in the adjuvant-induced arthritic rats (data not shown).

Although the levels of expression of osteoclastic cells and bone erosion in the joints progressed with time in both control and C60-treated groups, the C60-treated group showed significantly lower levels at four weeks and six weeks to the respective control group (Figures $3 \mathrm{~A}, \mathrm{~B}, \mathrm{C}$ ).

C60 had suppressive effects both on osteoclast differentiation and bone resorption, which leads to the possibility of C60 being beneficial in osteoporosis. RA patients often have juxta-articular osteoporosis as well as systemic osteoporosis. ${ }^{1-3}$ Our findings suggest that C60 is expected to exert a beneficial effect on juxta-articular osteoporosis by dual mechanisms, both direct and indirect. One is a direct suppressive effect on osteoclast differentiation in that C60 inhibits the RANK-RANKL signal by removal of ROS, a messenger of the signal pathway. Recent studies have clearly revealed that ROS mediates RANK-RANKL signaling in osteoclastogenesis. ${ }^{9-15}$ We confirmed in vitro that C60 significantly suppressed osteoclast differentiation in vitro.
This suppressive effect is considered to be exerted at the step of differentiation of precursor cells into osteoclasts. Another is the indirect or secondary effect exerted by suppressing the production of proinflammatory cytokines which can enhance the intensity of the RANK-RANKL signal. TNF- $\alpha$, IL-1, IL-6, and IL-17 are also called bone resorptive cytokines and are known to accelerate bone resorption. ${ }^{22}$ There is general consensus that ROS is increasingly produced from inflammation-related cells in parallel with the stimulation by proinflammatory cytokines such as TNF- $\alpha$, IL-1, IL-6, and IL-17 and plays an important role as a messenger of the intracellular signal pathways. ${ }^{26,27}$ The present study sought to demonstrate in vitro and in vivo that water-soluble C60 fullerene can function as a protective agent against the bone resorption/destruction occurring in arthritis. Our in vitro studies indicate that water-soluble C60 inhibits the differentiation of osteoclast precursor cells into osteoclasts and osteoclastic bone resorption.

In conclusion, our findings indicate that water-soluble C60 fullerene can function as a protective agent against 
A

6 weeks

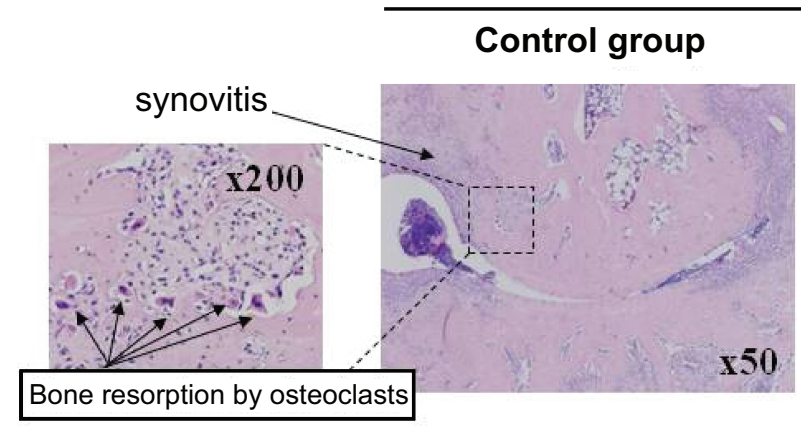

C60-treated group

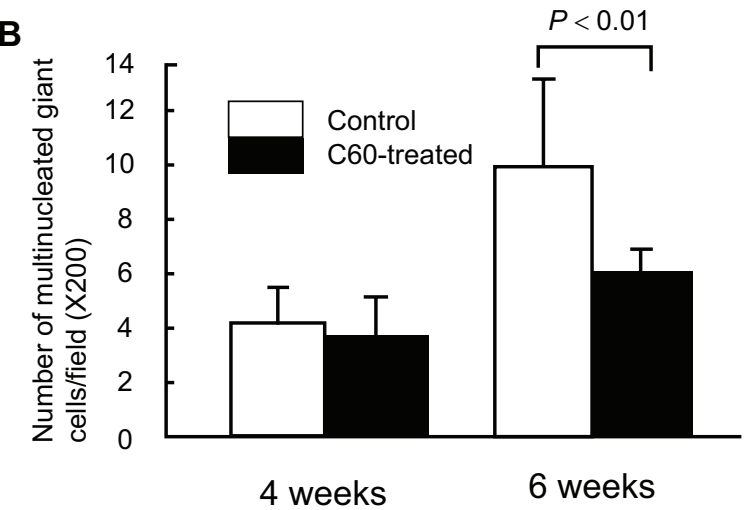

C

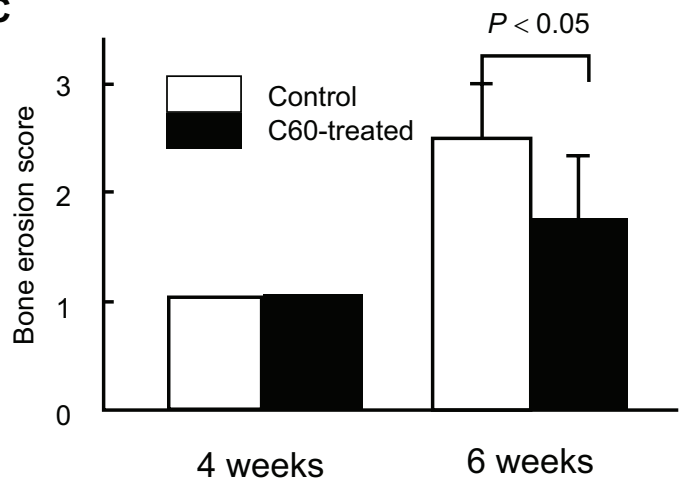

Figure 3 Intraarticular treatment of C60 inhibits the osteoclastic bone resorption in the arthritis model rats. A) As shown in the representative images of the joint damage at the six-week time point in arthritis model rats, joints that had been treated with phosphate-buffered saline showed severe joint destruction and bony ankylosis. In contrast, inhibitory effects were observed following administration of C60 in the C60-treated group. B) The expression of osteocalstic cells was histologically evaluated in each ankle joint sample (four rats per time point). Although the expression of osteoclastic cells in the joint progressed with time in both control and $\mathrm{C} 60$-treated groups, the $\mathrm{C} 60$-treated group showed significantly lower expression of osteoclastic cells at four and six weeks compared to the respective control group. C) The degree of bone erosion was histologically scored using the bone erosion score. Bone destruction was graded from 0 to 3 , where $0=$ normal, I = mild loss of cortical bone at few sites, $2=$ moderate erosion of cortical and trabecular bone, and $3=$ marked destruction of bone at many sites. The C60-treated group showed significantly lower bone erosion at four and six weeks compared to the respective control group.

osteoclastogenesis and the resultant bone hyper-resorption in vitro. Intra-articular treatment with this compound significantly reduces osteoclastic bone destruction in the rat model of arthritis. These findings suggest that C60 fullerene may be useful as a therapeutic agent for bone resorption in arthritis.

\section{Disclosures}

The authors report no conflicts of interest in this work.

\section{References}

1. Rooney M, Condell D, Quinlan W, et al. Analysis of the histologic variation of synovitis in rheumatoid arthritis. Arthritis Rheum. 1988;31:956-963.

2. Cush JJ, Pietschmann P, Oppenheimer-Marks N, Lipsky PE. The intrinsic migratory capacity of memory $T$ cells contributes to their accumulation in rheumatoid synovium. Arthritis Rheum. 1992;35:1434-1444.

3. Firestein GS. Rheumatoid synovitis and pannus. In: Klippel JH, Dieppe PA, editors. Rheumatology. London, UK: Mosby; 1998. p. 1-24.

4. Takayanagi H. Mechanistic insight into osteoclast differentiation in osteoimmunology. J Mol Med. 2005;83(3):170-179.

5. Sato K, Takayanagi H. Osteoclasts, rheumatoid arthritis, and osteoimmunology. Curr Opin Rheumatol. 2006;18(4):419-426.
6. Walsh MC, Kim N, Kadono Y, et al. Osteoimmunology: interplay between the immune system and bone metabolism. Annu Rev Immunol. 2006;24:33-63.

7. Takayanagi H. Osteoimmunology: shared mechanisms and crosstalk between the immune and bone systems. Nat Rev Immunol. 2007;7(4):292-304.

8. Alnaeeli M, Park J, Mahamed D, Penninger JM, Teng YT. Dendritic cells at the osteo-immune interface: implications for inflammationinduced bone loss. J Bone Miner Res. 2007;22(6):775-780.

9. Lean JM, Davies JT, Fuller K, et al. A crucial role for thiol antioxidants in estrogen-deficiency bone loss. J Clin Invest. 2003;112(6):915-923.

10. Ha H, Kwak HB, Lee SW, et al. Reactive oxygen species mediate RANK signaling in osteoclasts. Exp Cell Res. 2004;301(2):119-127.

11. Bai XC, Lu D, Liu AL, et al. Reactive oxygen species stimulates receptor activator of NF-kappaB ligand expression in osteoblast. J Biol Chem. 2005;280(17):17497-17506.

12. Lee NK, Choi YG, Baik JY, et al. A crucial role for reactive oxygen species in RANKL-induced osteoclast differentiation. Blood. 2005;106(3):852-859.

13. Yip KH, Zheng MH, Steer JH, et al. Thapsigargin modulates osteoclastogenesis through the regulation of RANKL-induced signaling pathways and reactive oxygen species production. J Bone Miner Res. 2005;20(8):1462-1471.

14. Varanasi SS, Datta HK. Characterisation of cytosolic FK506 binding protein 12 and its role in modulating expression of Cbfa1 and osterix in ROS 17/2.8 cells. Bone. 2005;36(2):243-253. 
15. Kim HJ, Chang EJ, Kim HM, et al. Antioxidant alpha-lipoic acid inhibits osteoclast differentiation by reducing nuclear factor-kappaB DNA binding and prevents in vivo bone resorption induced by receptor activator of nuclear factor-kappaB ligand and tumor necrosis factor-alpha. Free Radic Biol Med. 2006;40(9):1483-1493.

16. Kroto HW, Heath S, O’Brien SC, Curl RF, Smalley RE. C60: Buckminsterfullerene. Nature. 1985;318:162-163.

17. Kratschmer W, Lamb LD, Fostiropoulos K, Huffman DR. Solid C60: a new form of carbon. Nature. 1990;318:162-163.

18. Krusic PJ, Wassermann E, Keizer PN, Morton JR, Preston KF. Radical reaction of C60. Science. 1991;254:1183-1185.

19. Dugan LL, Lovett EG, Quick KL, Lotharius J, Lin TT, O’Malley KL. Fullerene-based antioxidants and neurodegenerative disorders. Parkinsonism Relat Disord. 2001;7(3):243-246.

20. Tsuchiya T, Yamakoshi YN, Miyata N. A novel promoting action of fullerene $\mathrm{C} 60$ on the chondrogenesis in rat embryonic limb bud cell culture system. Biochem Biophys Res Commun. 1995;206(3):885-894.

21. Xiao L, Takada H, Maeda K, Haramoto H, Miwa N. Antioxidant effects of water-soluble fullerene derivatives against ultraviolet ray or peroxylipid through their action of scavenging the reactive oxygen species in human skin keratinocytes. Biomed Pharmacother. 2005;59:351-358.

22. Gharbi N, Pressac M, Hadchouel M, Szwarc H, Wilson SR, Moussa F. [60]fullerene is a powerful antioxidant in vivo with no acute or subacute toxicity. Nano Lett. 2005;5:2578-2585.
23. Yudoh K, Shishido K, Murayama H, et al. Water-soluble C60 fullerene prevents degeneration of articular cartilage in osteoarthritis via down-regulation of chondrocyte catabolic activity and inhibition of cartilage degeneration during disease development. Arthritis Rheum. 2007;56(10):3307-3318.

24. Haruna Y, Morita Y, Yada T, Satoh M, Fox DA, Kashihara N. Fluvastatin reverses endothelial dysfunction and increased vascular oxidative stress in rat adjuvant-induced arthritis. Arthritis Rheum. 2007;56(6): 1827-1835.

25. Helyes Z, Szabó A, Németh J, et al. Antiinflammatory and analgesic effects of somatostatin released from capsaicin-sensitive sensory nerve terminals in a Freund's adjuvant-induced chronic arthritis model in the rat. Arthritis Rheum. 2004;50:1677-1685.

26. Matsuzawa A, Saegusa K, Noguchi T, et al. ROS-dependent activation of the TRAF6-ASK1-p38 pathway is selectively required for TLR4mediated innate immunity. Nat Immunol. 2005;6(6):587-592.

27. Babbar N, Casero RA Jr. Tumor necrosis factor-alpha increases reactive oxygen species by inducing spermine oxidase in human lung epithelial cells: a potential mechanism for inflammation-induced carcinogenesis Cancer Res. 2006;66(23):11125-11130.
International Journal of Nanomedicine

\section{Publish your work in this journal}

The International Journal of Nanomedicine is an international, peerreviewed journal focusing on the application of nanotechnology in diagnostics, therapeutics, and drug delivery systems throughout the biomedical field. This journal is indexed on PubMed Central, MedLine, CAS, SciSearch ${ }^{\circledR}$, Current Contents ${ } /$ Clinical Medicine,

\section{Dovepress}

Journal Citation Reports/Science Edition, EMBase, Scopus and the Elsevier Bibliographic databases. The manuscript management system is completely online and includes a very quick and fair peer-review system, which is all easy to use. Visit http://www.dovepress.com/ testimonials.php to read real quotes from published authors. 